\title{
Structural analysis shows spliceosome-induced closure of an $\alpha$-helical super-helix in the proto-oncogenic splicing factor SF3b1
}

\author{
Debanjana Maji, Alan Grossfield and Clara L. Kielkopf \\ Email Contact: debanjana_maji@urmc.rochester.edu \\ Department of Biochemistry \& Biophysics, Center for RNA Biology, \\ University of Rochester School of Medicine, Rochester, NY
}

The SF3b1 splicing factor is a subunit of a multiprotein SF3b complex that is a checkpoint protein for spliceosome assembly. SF3b1 comprises a super-helix of repeating $\alpha$-helical units. In the context of the spliceosome, the SF3b1 $\alpha$-helical repeats bind a duplex between the spliceosomal U2 snRNA and the pre-mRNA branch point sequence, which ultimately offers an adenosine nucleophile for the splicing reaction. Several SF3b structures now are available, including human homologues in the isolated state, pre- $\mathrm{B}, \mathrm{B}$, or $\mathrm{B}^{\text {act }}$ spliceosome assemblies ${ }^{1}$, and yeast homologues in the $\mathrm{A}$, pre-B, B, or $\mathrm{B}^{\text {act }}$ spliceosome assemblies ${ }^{2}$. Acquired mutations of SF3b1 commonly recur among hematologic malignancies and cancers. Active "opening" of the SF3b1 torus by ATP-dependent RNA unwindases has been proposed to load SF3b1 on the U2 - BPS duplex and alter branchpoint choice in the presence of cancer-associated mutations. Yet, whether the SF3b1 superhelix opens or closes, and the underlying structural changes, have yet to be rigorously analyzed.

As a starting point, we compared the structures of human SF3b1 in the isolated SF3b particle (PDB ID: 5IFE) with a representative SF3b1-containing spliceosome (Bact stage, PDB ID: 6FF4). After superposing the C-terminal helical repeats, we found that the SF3b1 torus undergoes an overall rotation of approximately $45^{\circ}$ from the isolated particle to the context of the $B^{\text {act }}$ spliceosome. Despite this large conformational change, we saw minor changes in the twist and pitch of the helical repeats, ruling out evenly distributed changes. Instead, we observed large localized RMSD's and centroid-to-centroid distances of the SF3b1 helical repeats between the contexts of SF3b particle and the $\mathrm{B}^{\text {act }}$ spliceosome. This culminated in a clamp-like action, whereby the $\mathrm{N}$ - and $\mathrm{C}$-terminal SF3b1 regions enclosed the U2 - BPS duplex. These structural changes of the human $\mathrm{B}^{\text {act }}$ complex were similar for the pre-B and B-stage spliceosomes, as well as for the yeast homologue. RNA-induced displacement of the SF3b5 subunit, followed by rotation of an SF3b3 subunit that in turn bridges the SF3b1 termini, appeared to close the SF3b1 torus.

Altogether, we conclude that the SF3b1 torus undergoes significant closure, rather than opening, following incorporation into the assembling spliceosome. This conclusion has important ramifications for the mechanistic roles of ribonucleoprotein unwindases in spliceosome assembly, as well as the molecular consequences of cancer-associated SF3b1 mutations.

${ }^{1}$ PDB ID: 5IFE, 6AH0, 6AHD, 5O9Z, 5Z56, 5Z57, 5Z58, 6FF4

2PDB ID: 69G0, 5ZWM, 5NRL, 5ZWO, 5LQW, 5GM6 